Revista Digital Año 8. No 11 - Año 2017. -pág. 1--136

ISSN 1853-1393

Resistencia. Chaco. Argentina - 2017

\title{
LA PRÁCTICA DOCENTE QUE TRASCIENDE AL ÁMBITO DE LA CLASE. LAS POSICIONES QUE CONSTRUYEN LOS PROFESORES EN TORNO A LAS ACTIVIDADES QUE REALIZAN EN LA ESCUELA SECUNDARIA
}

TEACHING THAT TRASCENDS THE CLASS ENVIRONMENT. TEACHERS' VIEWS ABOUT THEIR ACTIVITIES AT SECONDARY SCHOOL

\author{
María Gloria Saucedoำ- María Teresa Alcalá2 - Horacio Ademar Ferreyra ${ }^{3}$
}

Fecha de recepción: 14-07-2017

Fecha de aceptación y versión final: 17-10-2017

\section{Resumen:}

En el artículo presentamos avances de un proyecto de beca de investigación que aborda la práctica docente desde las voces de los profesores de escuela secundaria.

El objetivo sustancial consiste en describir las actividades que los profesores realizan en la organización escolar, así como las posiciones que construyen y las relaciones que establecen en torno a las tareas que emprenden, a las responsabilidades y roles que asumen más allá del aula.

Trabajamos desde un abordaje cualitativo de carácter exploratorio-descriptivo utilizando como estrategia metodológica el estudio de casos. Los instrumentos de recolección de datos empleados son las entrevistas semiestructuradas (dirigidas a profesores de distintas disciplinas que conforman los departamentos del Nivel secundario) y el análisis documental, los cuales son triangulados metodológicamente.

Los resultados obtenidos permiten identificar que la práctica que cotidianamente realizan los docentes transciende el ámbito de la clase al involucrar un entramado de actividades y relaciones que la traspasa, que enmarcan y condicionan la tarea.

Aquí se construyen otras formas de desempeño de su tarea y "posiciones docentes" atravesadas por la reconfiguración institucional ligada a la incorporación de nuevos sectores de estudiantes en el marco de las configuraciones escolares y sociales emergentes. Se ponen en cuestión los supuestos educativos de la escuela tradicional y la tarea propia del docente que es "enseñar" que aparece como central, aunque claramente sobrecargada con actividades que requiere del trabajo de contención social hacia los sujetos con derecho a la educación secundaria y de la participación con la comunidad educativa en la organización escolar.

\footnotetext{
${ }^{1}$ Profesora en Ciencias de la Educación. Especialista en Análisis de la Educación; sus organizaciones y sus prácticas. Facultad de Humanidades-UNNE. Becaria de investigación. UNNE-CONICET. Correo electrónico: gloriasaucedo48@hotmail.com.ar

2 Profesora en Ciencias de la Educación. Facultad de Humanidades. UNNE. Magister en Tecnología de la Educación. Universidad de Salamanca. Directora del Instituto de Ciencias de la Educación. Facultad de Humanidades. UNNE. Docente e Investigadora en el campo de la Didáctica, el Curriculum y la Formación Docente. Correo electrónico: mtalcala@gmail.com ${ }^{3}$ Doctor y Licenciado en Ciencias de la Educación (Universidad Católica de Córdoba - UCC). Posdoctorado en Ciencias Sociales (Universidad Nacional de Córdoba, Centro de Estudios Avanzados, Argentina; Universidad Autónoma Metropolitana, Unidad Xochimilco -UAMx- México; Universidad de Oviedo España y Universidad Complutense de Madrid -España). Subsecretario de Estado de Promoción de Igualdad y Calidad Educativa del ME Córdoba. Docente e Investigador de la UCC. Unidad Asociada CONICET y de la Universidad Nacional de Villa María UNVM; docente e investigador invitado del Doctorado en Educación en la USTA (Colombia) y ITEC Guadalajara (México). Email: hferreyra@coopmorteros.com.ar
} 
Palabras Clave: Práctica docente. Escuela secundaria. Organización escolar. Posiciones docentes. Configuración escolar y social emergente.

\begin{abstract}
:
The progress of a research scholarship project is presented in this article. Teaching is approached from the perspectives of secondary school teachers. The main aim is to describe activities teachers do within the school organization and also the positions they build and also the relations they establish concerning the tasks they perform, the responsibilities and the roles they take beyond the classroom.

We used an explorative - descriptive qualitative approach with case study as the methodological strategy. The data collection procedures are: semi structured interviews (with teachers of different subjects belonging to the departments of a secondary school as participants) and documentary analysis methodologically triangled.

The results show that everyday teaching practice goes beyond the class environment since it involves an intertwined net of activities and relations that exceeds, frames and conditions it. Other ways of performing the teacher's task and "teachers' views," crossed by the institutional re-configuration due to the joining of new groups of students framed in the emerging school and social configurations, are built here.

Educational assumptions about traditional school and the teachers' own task, teaching, are questioned. "Teaching" comes up as central, though clearly overloaded with activities which require social containment work for individuals with the right to secondary level education and the participation of teachers together with members of the educational community in the school organization.
\end{abstract}

Key words: Teaching practice. Secondary school. School organization. Teachers' views. Emerging school and social configuration.

\title{
1-Nuestro punto de partida
}

El programa institucional con el que fueron pensadas las instituciones modernas en la función de instituir y socializar a los sujetos, a partir de la década de los '70, y sobre todo en el contexto de los 90 , ha entrado en crisis, se ha generado lo que Dubet (2010) llama el declive y lo que Tiramonti (2003) denomina proceso de desinstitucionalización. Este proceso afectó ra eficacia de las instituciones que regulan la vida y la existencia de los individuos: Estado, escuela, familia, trabajo; por un lado, modificando su estructura y función, y por el otro, estableciendo contradicciones y desorientaciones en los sujetos que se forman en ellas.

Este escenario que Pineau y Birgin (2006) llaman "estallido de las instituciones y de las subjetividades", sumado a las transformaciones que se han producido en la estructura social y el "golpe de timón" hacia políticas públicas de corte social que tuvo lugar tras la crisis del año 2001 en Argentina, enmarcan una nueva etapa de reposicionamiento estatal y de lineamiento de la política educativa nacional que establece como algunos de sus fines y objetivos en la Ley de Educación Nacional N²6206: el derecho a la educación, la inclusión social, la extensión de la obligatoriedad.

Dentro del marco del sistema educativo en general y en la escuela secundaria en particular, se genera una restructuración del sentido de la educación y de su funcionamiento, cuestionándose de este modo la idea originaria de la matriz escolar de la modernidad caracterizada por el carácter universal, único, hegemonizado y homogeneizante. Al respecto, identificamos en la legislación escolar la definición política expresada en la obligatoriedad para todos los ciudadanos e introduce la necesidad de la revisión ideológica y conceptual del origen histórico que impregnó su sentido. (Asprella, 2013, p. 25).

En la actualidad, se trabaja en una escuela secundaria para todos acompañada de otros formatos escolares que se vinculan con los mandatos sociales, aspiraciones, expectativas y demandas de la comunidad educativa, en donde los actores construyen su significado en un estado de interacción y tensión permanente entre el 
modelo anterior y la nueva constitución ante un cambio de época y realidad social y política, acontecimientos que nos llevan a un replanteo y reflexión en tres planos de análisis: macropolítica, mesopolítica y micropolítica.

Aquí nos centramos en las condiciones de la micropolítica de la escuela; formatos escolares, laborales, entre otros, que hoy se presentan y en consecuencia transforman o crean "nuevas cotidianeidades", tanto institucionales como de construcción de subjetividad individual y colectiva. (Asprella, 2014, p.30). Nos interesa describir el modo en que se desempeña la práctica docente que transciende al ámbito de la clase adquiriendo nuevas configuraciones en los contextos sociales y escolares emergentes, donde los profesores realizan tareas más allá del salón de clases, así como identificar las posiciones que construyen y las relaciones que establecen en su trabajo cotidiano.

Si bien, como resultado de una tradición organizativa y administrativa, se entiende a la práctica docente como un trabajo individual que se realiza en el aula, en este escrito, la abordamos desde la complejidad como un trabajo colectivo que se enfrenta a una práctica y cultura escolar establecida y como una configuración "dada" del espacio de trabajo del profesor.

Es decir, reflexionamos sobre la práctica docente desde tiempos y procesamientos individuales y también colectivos, enmarcada en una organización escolar con ritmos y apoyos institucionales particulares, en los que suponemos se presentan situaciones problemáticas, desafíos y responsabilidades que se expresan en los tipos de actividades desarrolladas, las tareas y roles que asumen como las posiciones que construyen y relaciones que establecen los profesores en torno a ella.

En este sentido nos preguntamos: ¿Qué dicen los profesores sobre las actividades que realizan en la escuela? ¿Cómo se establecen las tramas de relaciones entre los docentes, estudiantes y tutores en el accionar cotidiano? ¿Cuáles son las posiciones que los profesores construyen, los desafíos y responsabilidades que conlleva la institucionalización del trabajo colectivo en la organización escolar en el contexto de las configuraciones escolares y sociales emergentes?

\section{2- El abordaje metodológico}

\subsection{Definición y justificación de la perspectiva metodológica}

Abordamos el campo de estudio desde una metodología de diseño cualitativo de carácter exploratorio-descriptivo, mediante la descripción y análisis del proceso utilizando como estrategia metodológica el estudio de casos.

Está sustentado desde una lógica de la perspectiva hermenéutica; una orientación epistemológica y teórico-metodológica para indagar, comprender e interpretar las características y la situación actual del objeto de estudio desde las "voces" de los profesores, cómo cada uno de ellos viven y resignifican la práctica docente en la organización escolar en relación con las actividades y tareas que realizan y los roles y responsabilidades que asumen, así como, las interacciones entre los actores y las posiciones que construyen en torno a ellas en el contexto histórico, legal, político, cultural e institucional que la circunscribe.

Considerando la lógica dialéctica-compleja en el abordaje de la relación trabajo teórico -categorías conceptuales- y trabajo empírico -significados y puntos de vista de los actores-, se recurrió en términos de Morín (1986), a un análisis de la complejidad y multirreferencial.

En este sentido, abordamos el objeto de estudio desde la perspectiva teórica de la política educativa (análisis de los planos macropolitico, mesopolitico y micropolitico de las normativas y las resoluciones vigentes, etc.), con aportes de la pedagogía y la sociología de la educación (práctica docente, roles y tareas, función de 
la escuela, perspectiva micropolítica: cultura institucional y organizacional, entre otros) y lo analizamos desde la categoría teórica-empírica de la organización escolar: actividades de los docentes en los órganos de participación y gestión, actividades de los docentes con los tutores y actividades de los docentes con los estudiantes.

\subsection{Universo de estudio, unidad de análisis y muestra}

La institución educativa se encuentra ubicada en el casco céntrico de la capital, en la provincia de Corrientes. Presta servicio a una población de 450 estudiantes y se caracteriza por ser una escuela de Nivel secundario que por un período significativo ha sido anexo de otro establecimiento educativo y que hace seis años se ha independizado del mismo.

La población en estudio está constituida por los profesores de enseñanza secundaria. Con la intención de caracterizar, desde las voces de los profesores, las actividades desarrolladas en la escuela, se seleccionó una muestra de 21 profesores de 25 a 50 años de distintos espacios curriculares que conforman los Departamentos que responden a la particularidad de la oferta educativa de la escuela secundaria.

\subsection{Componentes del diseño metodológico e instrumentos de relevamiento de la información}

Hemos considerado relevante el empleo de dos instrumentos de recolección de datos: entrevistas semiestructuradas y observación documental.

En primer lugar, para abordar el objeto de estudio realizamos entrevistas semiestructuradas a los docentes; 6 jefes de Departamento correspondientes a: Lengua y Comunicación, Matemática, Ciencias Exactas y Naturales, Lenguaje Artístico y Educación Física, Ciencias Sociales, Informática y Fortalecimiento de las Trayectorias Escolares; y a 15 docentes de las siguientes asignaturas: Matemática, Lengua y Literatura, Inglés, Teatro, Educación Artística, Química, Ciencias Sociales, Física, Informática, Educación Física, Biología y Psicología.

Los profesores entrevistados manifestaron sus opiniones sobre las actividades y tareas que realizan fuera del aula, los roles, las responsabilidades que asumen, así como sus opiniones convergentes y divergentes sobre las experiencias realizadas en la institución educativa.

En segunda instancia, con la intención de completar y confrontar la información recopilada por medio de las entrevistas semiestructuradas, recurrimos al instrumento de la observación documental (Ley de Educación Nacional № 26206, resoluciones nacionales y jurisdiccionales, circulares institucionales, planificación de actividades) con la finalidad de analizar la normativa que da marco a la materialización de la práctica docente en el establecimiento educativo en referencia al campo de estudio.

\subsection{La estrategia y el proceso de análisis de los datos}

Considerando las distintas descripciones detalladas y recopiladas de las interacciones, como los extractos de los documentos realizamos una triangulación metodológica entendida como la combinación en un estudio único de distintos métodos o fuentes de datos (Taylor y Bogdan, 1986, p. 92) mediante la práctica intramétodo (Mendicoa, 2003,p.75), que favoreció una comprensión e interpretación más abarcativa del objeto en estudio y de las variables, lo que permitió someter los datos a control recíproco. 


\section{3-Desandando las categorías teóricas: Las prácticas y posiciones docentes en el marco de las configuraciones escolares y sociales emergentes}

En el contexto de la sanción del derecho de la educación secundaria, la inclusión social, la obligatoriedad escolar y la universalización del nivel, entendemos a las configuraciones escolares y sociales emergentes a las organizaciones educativas, donde los supuestos de la escuela tradicional son actualizados por la práctica cotidiana de los actores hacia la apertura de nuevos sectores sociales.

Según Marmissolle (2016) quien aborda las configuraciones escolares, se trata de un entramado organizacional complejo, variable y cambiante donde interactúan jóvenes y adultos que se caracterizan por actualizar y resignificar las formas de ser estudiante, docente y/o directivo, se plantean nuevas demandas, desafíos e incertidumbres respecto a las condiciones laborales de los actores educativos y a las finalidades de la escuela que comienzan a ser cuestionadas frente a la incorporación de nuevos sectores.

Las fronteras que delimitan el espacio institucional están fuertemente permeadas por las condiciones sociales y culturales de la comunidad del que la escuela forma parte y por las tensiones de las que los jóvenes escolarizados son portadores, quienes en años anteriores han sido excluidos de la educación secundaria. Sin embargo, en la actualidad se plantea como un desafío construir y negociar con estos nuevos sujetos, resguardarlos, así como modificar los lazos que se crean entre éstos y los actores educativos (docentes, directivos, supervisores, etc.).

En este sentido, entendemos que el habitus que funciona como inconsciente colectivo, es decir, de ideas compartidas por los adultos que forman parte de la configuración escolar y que organizan su cotidianeidad, se ve fracturado en sus supuestos organizadores porque la matriz con que se formó se asentó en la relación de los docentes con unos sujetos sociales convertidos en alumnos por su presencia y voluntad de asistir a la escuela, y eventualmente aprender algo. (Marmissolle, 2016, p. 184).

Los supuestos teóricos y políticos que sostuvieron la formación y actuación de los profesores como las condiciones del ejercicio cotidiano de la prácticas docente en el aula, ya no se resumen en las convencionales relaciones pedagógicas destinadas a garantizar cierto modo de abordar los procesos de enseñanza y aprendizaje de los contenidos curriculares, sino también resignifican sus obligaciones y actividades en la organización escolar, en el marco de las demandas y los desafíos que generan el trabajo colaborativo y participativo en contextos escolares y sociales emergentes.

En este marco de desenvolvimiento cotidiano e identidades sociales propias se desarrolla la prácticas docente. Aquí nos interesa identificar las relaciones entre los actores escolares como miembros de la organización escolar y las condiciones que influyen e intervienen en el funcionamiento de ésta. Es decir, cómo los docentes significan sus obligaciones y prácticas, qué tareas y responsabilidades les genera desempeñar su rol en el marco de las configuraciones escolares y sociales emergentes.

Para abordar esta idea, recuperamos la conceptualización presentada por Fierro (2005) quien señala que la práctica docente:

"es una praxis social, objetiva e intencional en la que intervienen los significados, las percepciones y las acciones de los agentes implicados en el proceso -maestros, alumnos, autoridades educativas y padres de familia- así, como los aspectos políticos institucionales, administrativos y normativos que, según el Proyecto Educativo de cada país, delimitan la función del maestro". (p. 21). 
En relación con la definición planteada por la autora, consideramos que las relaciones entre los actores educativos se entremezclan formando una trama de actividades y tareas, en ocasiones, difíciles de comprender por la sobrecarga que implica al trabajo del profesor y por lo que consideramos que la práctica docente trasciende la tarea de la enseñanza en el aula.

Como argumento central, parafraseamos la conceptualización planteada por Achilli (1986) destacamos la complejidad y el carácter situacional de la práctica docente al definirla como el trabajo que el profesor desarrolla cotidianamente en determinadas y concretas circunstancias sociales, históricas e institucionales que tensan acontecimientos objetivos y subjetivos de la misma y que adquiere significaciones particulares para los actores educativos y la sociedad. Trabajo que si bien está definido en su significación social y particular por la práctica de enseñanza, va más allá al involucrar una entramada red de actividades y relaciones que la traspasa, que enmarcan y condicionan la tarea, que se entrecruzan con los rasgos constitutivos de los profesores como las posiciones que construyen en vinculación a ella.

Esas tareas, que superan la práctica pedagógica, van desde aquellas que podemos caracterizar como burocráticas (planillas, planificaciones, preparación de la documentación del alumnado, etc.) hasta las que se desarrollan en la organización escolar, pasando por las reuniones de personal con directivos, asesoramiento y reuniones con padres, así como, las ventas de rifas, cobro de cuotas cooperadoras de padres, etc.

Estas actividades y relaciones que cotidianamente el docente despliega "fuera" del aula y que son vividas por él como una sobrecarga de tarea, perturban la práctica pedagógica concreta -en su especificad de trabajo intelectual- al producir un distanciamiento de la misma. Es decir, la especialidad del trabajo del profesor queda desdibujada e incorporada fragmentariamente a un conjunto de funciones que si bien pueden suponerse como secundarias en el desempeño del rol docente, no ocurre así en el contexto burocrático de la organización institucional del que forma parte. (Achilli, 1986, p.7).

Sin embargo, estas características que adquiere la prácticas docente se enriquecen u obstaculizan en relación con el desarrollo de las prácticas escolares alternativas y con la particularidad de la cultura organizacional, dado que éstas proponen un modo de relacionarse y desempeñarse en la escuela generando una ruptura con el formato escolar tradicional y/o dando paso a nuevas formas de entender el trabajo colaborativo y participativo en la organización de la escuela.

En virtud de lo expuesto, podemos decir, que los rasgos del trabajo del docente y la cultura escolar distinguen a la escuela secundaria de otras instituciones e instancias de socialización que configuran su identidad, autonomía y modo de concebir "el trabajo colectivo y participativo". Es decir, cómo los profesores perciben, entienden y viven las prácticas docentes en la organización escolar, y cuáles son las posiciones que construyen en torno a ellas, que históricamente se constituyeron bajo la tradición organizativa y administrativa como un trabajo individual y en el aula.

En el presente artículo, tal como mencionamos en los párrafos precedentes, abordamos la práctica docente desde la complejidad como un trabajo colectivo que se enfrenta a una cultura establecida y a una configuración "dada" que requiere del apoyo institucional. Al respecto sostenemos que "cuando se trata de abordar las prácticas es ineludible plantearse el problema de la organización porque en ella radica la generación de condiciones para buscar nuevas formas de trabajo". (Elmore citado en Ezpeleta, 2004, p.419).

Desde esta mirada de la complejidad y del carácter situacional sobre la práctica docente, aludimos a la conceptualización y caracterización de las posiciones docentes referentes al trabajo de enseñar en la escuela secundaria. 
En palabras de Southwell y Vassiliades (2014) los sujetos (profesores) construyen en su trabajo cotidiano posiciones docentes que suponen la configuración de identidades que organizan relaciones, dinámicas y estrategias a partir de concepciones específicas respecto del sentido de su tarea que se instituyen históricamente desde los significados de los diferentes actores que se desempeñan en la tarea.

Al recuperar la conceptualización de estos autores, entendemos que las posiciones se componen de la circulación de sentidos y de discursos que regulan y organizan el trabajo de enseñar en el aula. Es decir, las concepciones especificas respecto del sentido de su tarea, la presencia de elementos históricos de la profesión, y los múltiples modos en que los sujetos enseñantes asumen, viven y piensan su tarea, ha sido configurado desde la práctica de la enseñanza. Sin embargo, en este artículo, la resignificamos en relación con las prácticas docentes desde la mirada organizacional.

En este sentido, sumamos uno de los dos elementos de la conceptualización dada por los autores Southwell y Vassiliades (2014) que desarrollan Birgin y Pineau (2014) sosteniendo que la posición docente supone la construcción histórica y social de miradas acerca de los problemas educacionales a los que se enfrentan; se trata de sedimentaciones en el tiempo de elementos que se rearticulan en el presente, configurando así nuevas formaciones. (Vassilliades, 2012 citado en Birgin y Pineau, 2014, p.3).

En esta línea de trabajo que nos proponen los autores anteriormente señalados, nos interesa identificar algunos aspectos de las cuatro posiciones que plantean Birgin y Pineau (2014) en relación con los cambios que se produjeron en la formación docente y en la historia de la escuela secundaria en Argentina: aristocracia profesoral, profesores diplomados, profesores como profesionales de la sociedad salarial y profesores ante los efectos de la fragmentación educativa. Aunque claramente nos centraremos en algunos rasgos de la última posición docente que sobre lo contextual coinciden con las características de las configuraciones escolares y sociales emergentes presentadas en este escrito.

\section{4- Las posiciones docentes que construyen los profesores en torno a las actividades en la organización escolar. Tareas y roles asumidos}

Enmarcándonos en el contexto sociopolítico y en un escenario en que el sistema educativo en general y el nivel secundario en especial, vuelven a ser objeto de priorización de las políticas públicas, identificamos en la normativa algunos indicios que promueven los espacios institucionales de participación de los actores educativos como "comunidad de trabajo"; lo que conlleva revisar las prácticas pedagógicas y de organización escolar, es decir, se propone una mirada reflexiva y participativa "desde el aula y desde la escuela". (Ferreyra y Vidales, 2012, p. 38).

En este sentido, analizamos las prácticas docentes desde la mirada de la organización escolar considerando tres ejes: actividades de los docentes en los órganos de participación y gestión, actividades de los docentes con los tutores y actividades de los docentes con los estudiantes.

\subsection{Organización escolar: actividades de los docentes en los órganos de participación y gestión}

En la práctica cotidiana, según los entrevistados, participan en la elaboración de los acuerdos de convivencia, en el reglamento interno y en el Proyecto Educativo Institucional como lo establece la normativa, aunque admiten que las convocatorias y los modos de organización se realizan, en algunas ocasiones, de modo esporádico y 
confuso, y otras, abiertas al diálogo y a la generación de propuestas en el marco de las reuniones institucionales que se realizan a principio y durante el transcurso del año escolar.

En términos de Birgin y Pineau (2014) la responsabilidad y la posición docente asumida en la escuela, está vinculada con los principios y fines de la política educativa planteada desde las legislaciones educativas de los 90 hasta la actualidad, en los que se establece como derechos y obligaciones, la activa participación en la elaboración e implementación del proyecto institucional de la escuela y en el gobierno de la educación por sí y/o a través de sus representantes.

Al respecto sostenemos que la tarea que conllevaba el Estado de control de la implementación de las políticas educativas y pedagógicas, en la actualidad, plantea una responsabilidad delegada y compartida por la comunidad educativa. Ahora, los profesores tienen el compromiso de asumir la tarea de participar en las propuestas de construcción de proyectos educativos, en la asociación de cooperadora, en la constitución de los acuerdos escolares de convivencia escolar, entre otros, sumando a la tarea que conlleva la práctica de la enseñanza en el aula.

Para ilustrar la afirmación teórica y empírica recuperamos un fragmento de la entrevista realizada a la jefa de Departamento de Matemática quien sostiene:

El acuerdo de convivencia se está trabajando todavía, porque el acuerdo de convivencia no está totalmente establecido todavía acá. Se está trabajando. Acá están trabajando psicopedagogos con los profesores y tuvimos reunión institucional para trabajar sobre ese tema. Porque al desprenderse esta escuela del XXXX, entonces tenemos que organizarnos, se está organizando y el acuerdo de convivencia y el PEI todo eso se está trabajando.

La cooperadora ya está, ya se organizó la cooperadora. Está todo bien organizada la cooperadora. El reglamento interno, el acuerdo de convivencia trabajamos primero en una reunión institucional se hizo la bajada y después se van haciendo reuniones pero la que coordina es la psicopedagoga y los profesores de tutoría son los que están XXXX. Pero trabajamos conjuntamente con todos los grupos, las áreas, los departamentos.

Tal como menciona la docente entrevistada en la institución educativa todavía se está organizando con la participación de la comunidad la normativa interna, aunque también identificamos desde las voces de los profesores de escuela secundaria, por la estructura, organización, espacios, tiempos y subjetividades que caracterizan y condicionan su trabajo, ven obstaculizada la implementación en referencia al sentido original de la propuesta desde la normativa. Podríamos decir "los docentes resuelven las tareas como pueden". Al respecto una docente señala: "se participa pero yo creo que capaz no lo suficiente porque cada uno tiene su actividad, la mayoría tienen varias escuelas y demás motivos."

\subsection{Organización escolar: actividades de los docentes en relación con los tutores}

Según los docentes el contacto con las madres y los padres de los estudiantes se da principalmente a través de las reuniones de entrega de libretas o reuniones específicas con los tutores de aquellos alumnos que tienen problemas de disciplina.

Aquí parecería que la escuela reduce a este tipo de actividades y sostiene una idea de participación de las familias en el ámbito escolar vinculadas a la escucha de la información sobre "el registro académico" que se ofrece a través de la entrega de 
libretas y a la indisciplina de aquellos "sujetos que no se ajustan a la norma". De este modo, se estaría reflejando una relación construida históricamente entre los tutores y la escuela. (Ojeda y Saucedo, 2015).

En este sentido, podríamos afirmar en palabras de Martínez Cerón (2005), los planteamientos sobre la participación de las familias en la organización escolar establecida en el marco normativo y en las distintas resoluciones, entra en contradicción con los supuestos que los docentes han construido en su formación y en modelos escolares cerrados que no han cambiado en lo esencial.

Podríamos decir, en palabras de Ojeda y Saucedo (2015) las concepciones relacionadas con el coprotagonismo de las familias en la escuela, desde las "voces" de los docentes quedan circunscriptas a la participación desde el nivel informativo y vinculado con el rendimiento y comportamiento escolar de los estudiantes, en las que ambos actores; profesores y tutores "se acompañan y se apoyan" comprendida como una actividad compartida, colaborativa y de dependencia, asumiéndose como positiva.

También el acercamiento y el diálogo entre estos actores se establecen en relación con la comunicación por la inasistencia en la escuela, así como las orientaciones en las distintas problemáticas de sus hijos. Por ejemplo: "el hijo no quiere venir más a la escuela (...) no hay acompañamiento de los padres." Al respecto una docente manifiesta: (....) Pero a veces ellos mismos con todos sus problemas encima los tutores no saben cómo manejar la situación con los hijos y bueno lo que tratamos desde nuestro lugar es orientarlos un poquito."

Asimismo, desde las voces de los profesores, la vinculación con los tutores radica en el acompañamiento y el apoyo por el rendimiento escolar y la formación de los estudiantes. Aquí comprendemos que la posición del docente en relación con su tarea implica no solo el seguimiento al estudiante en el aula, sino también, en sentido amplio y considerando su contexto, propone la vinculación con los tutores "el apoyo hacia y desde" cada uno de los actores.

También, identificamos en la figura de los docentes el rol de "mediador", intermediario entre los alumnos del ciclo superior y los tutores en el acompañamiento de las actividades escolares y en la elección de la carrera de grado. Al respecto una profesora manifiesta: "Siempre el docente está en medio como mediador entre el alumno y la escuela porque van y vienen, el mensaje va y viene, y uno ve la respuesta (...)".

También, se consideran "mediadores" entre lo que se propone en el régimen de convivencia por ejemplo el uniforme y la realidad socioeconómica de los estudiantes.

En referencia al rol de mediador que caracteriza al docente, recuperamos un fragmento de la entrevista realizada a la jefa del Departamento de Matemática que expone lo siguiente:

"siempre el docente es el intermediario. Vos háblale a tu mamá, a tu papá explícale tal cosa y vez después la actitud del alumno. Es decir, que uno está constantemente intermediando. Habla con tu mamá, habla con tu papá."

"Pero yo creo que en ese sentido escuela, docente y la familia. El docente es como mediador porque tenemos que también cuidar a la escuela, pero también somos personas, vemos a las familias desde otro punto de vista. Yo me pongo como mediadora a veces, hay que buscar el equilibrio que es lo que más cuesta." 


\subsection{Organización escolar: actividades de los docentes en relación con los estudiantes}

Los docentes entrevistados plantean la necesidad de contribuir con las tareas de "contención económica, afectiva y social hacia los estudiantes", y también, sostienen que es el sentido y la demanda que se les plantea desde la comunidad educativa.

Al respecto caracterizamos esta idea desde las voces de algunos docentes entrevistados:

"Lo que hacemos es indagar nosotros primero con los profesores tutores, indagar el problema, informar al directivo, informar a los profesores $y$ tratar de contener al chico primero tenemos que contenerlos. Y también así a las familias que por ahí necesitan, orientamos a las familias". (Entrevista docente del Departamento de Fortalecimiento de las Trayectorias Escolares)

"(...) Por ahí los padres vienen y lloran y bueno. Ahí entro yo otra vez, le saco aparte y le digo a vos te gusta que tu mamá esté llorando, $X X X X$ te crio bien y vos le estas fallando, cumplo con la realidad social otra vez." (Entrevista jefe de Departamento de Lenguaje Artístico y Educación Física)

Las afirmaciones planteadas por los docentes son comprendidas en el marco de la incorporación de nuevos sectores sociales en la escuela secundaria, con trayectorias escolares, tradiciones y capitales culturales diferentes para lo que fue pensado el nivel medio, desde la voces de los trabajadores de la educación, se instala la necesidad que los profesores cumplan con la tarea de enseñar, pero a su vez, se comprometan con la tarea social de contener y acompañar a los estudiantes.

“(..) por ahí vienen los papás dicen que el chico anda mal, que no quiere estudiar, que le cuesta venir a la escuela, cosas así, que le ven muy triste, que no quieren hacer los trabajos que les mandan los profesores". (Entrevista docente del Departamento de Fortalecimiento de las Trayectorias Escolares)

Al respecto recuperamos los aportes de Southwell y Vassiliades (2014) quienes expresan la noción de posición docente vinculada por un lado, con la enseñanza como acto político en el que el desafío según los docentes consiste en "ser educador, acompañar a los chicos y guiarlos como educador", y por otra parte, a la relación que se construye con los otros sobre qué hacer y qué papel desempeñar en relación con la sociedad, es decir, "estar comprometidos con la realidad escolar y con la actividad escolar."

En este marco, tal como señalan los autores la construcción de la posición docente implica formas de sensibilidad, interpelación y prefiguración de vínculos en relación con las situaciones y con los "otros". Según los relatos de los trabajadores de la educación, se interesan por indagar sobre el problema de los alumnos, informar a los preceptores, directivos, y algunas veces, a los tutores. Se podría decir, están comprometidos con la realidad y la actividad escolar desde su complejidad y en relación con todos los actores que intervienen en la acción educativa. Al respecto una docente expresa:

"Acá los chicos de sexto por ejemplo que ellos se compran las chombas de egresado (...) No todos tienen posibilidades de tener, pero 
uno les habla con ellos y trata de colaborar de alguna manera (...) Y trabajamos y pusimos algunos profesores para que el alumno que no se pudo comprar entre todos pudiéramos."

Aquí identificamos algunos rasgos de la cuarta posición docente planteada por Birgin y Pineau (2014) que aborda el trabajo de los profesores ante los efectos de la fragmentación educativa y ubica en un período de importantes transformaciones económicas, sociales y culturales que involucran de modo directo a las escuelas y a los docentes.

Los autores manifiestan que en las últimas décadas, bajo los efectos de la "fragmentación social" y los profundos cambios socioculturales, se construyeron otras formas de desempeño. Estos cambios no solamente afectaron las variables estructurales y laborales sino también instauran "nuevas cotidianeidades", por ejemplo en el quehacer de la práctica docente. Es decir, si bien se destaca como tarea prioritaria de los docentes, la clase, advertimos en sus expresiones la importancia que para ellos tienen la contención de los alumnos y el compromiso social que asumen en la mediación de la formación de estos sujetos.

En este contexto, se le demanda al docente que contenga y acompañe a los estudiantes, que adquiera un rol de mediador "entre él y los tutores, entre él y las nuevas formas de vida social de los alumnos". En palabras de Asprella (2013) redefine lo social a partir de nuevas y múltiples categorías o sujetos sociales, nuevas necesidades y heterogeneidad de situaciones.

Podríamos sostener que la tarea que asume el profesor está dirigida a satisfacer las necesidades más inmediatas, como contención afectiva o apoyo en relación con nuevas problemáticas juveniles (...) la atención para concentrarse en la inmediatez de sus necesidades. (Sendón, 2007, citada en Aprella, 2013, p.28)

De este modo, creemos que la tarea de los docentes en algunas actividades que se proponen desde la escuela, no están alejadas de la cuestión social. (Asprella, 2013, p. 28). Además, de "la colaboración económica", se destaca "la solidaridad y el compromiso social", que asumen los docentes para acompañar a los alumnos no solo en las cuestiones académicas, sino también, en aquellas vinculadas con la integridad psicológica y social de éstos. Por ejemplo: colaborar con los estudiantes de escasos recursos económicos en la compra de la indumentaria para la finalización del ciclo superior, así como, en casos que las familias y los alumnos necesiten. Al respecto una docente señala: "(...) se quemó la casa hubo un incendio, y entonces, si hay grupos de profesores que si nos acercamos, tratamos de colaborar con esa familia."

\section{5-Reflexiones finales}

Tal como afirma Birgin (2007) cuando las políticas macro y microinstitucionales se proponen producir transformaciones $u$ otros modos de mirar la escuela y la docencia, las acciones de los profesores se comprenden en un proceso de negociación con tradiciones, historias y culturas que constituyen a éstos en su tarea de enseñar, que a su vez, en un contexto de fragmentación social y de un programa institucional en declive (Dubet, 2010, p.7) requiere pensar en «nuevas posiciones docentes » de enseñanza secundaria, en herencia, oposición, continuidad y novedad. (Birgin y Pineau, 2014, p.60).

Pensar en "el nuevo sentido de la escuela secundaria" que propone cambiar o crear en términos de Asprella (2013) "nuevas cotidianeidades", tanto institucionales como de construcción de subjetividad individual y colectiva generando incertidumbres en el quehacer de la cotidianeidad escolar. 
Es decir, en este escenario nos situamos en un espacio de incertidumbres entre las demandas de las políticas del sistema educativo, nuestras ideas sobre la enseñanza y el para qué del ejercicio y la práctica docente, donde algunos rasgos de la forma escolar moderna continúan en algunas construcciones identitarias del trabajo docente, mientras otros actores producen subjetividades, prácticas e identidades que emergen, creando incipientes formas de interactuar en las organizaciones escolares.

Advertimos en los testimonios de los profesores que la práctica que cotidianamente realizan los docentes trasciende la tarea de enseñar vinculándose más con las exigencias del contexto de las configuraciones escolares y sociales donde los supuestos educativos de la escuela tradicional son actualizados por la práctica cotidiana de los actores en la organización de la institución educativa.

Al respecto, advertimos que la tarea de "enseñar" aparece como central aunque claramente sobrecargada con tareas de contención social y acompañamiento de los estudiantes, acciones que implican distintas actividades como la obligación de interactuar con la comunidad educativa que conlleva al trabajo colaborativo con los tutores y colegas, así como la participación en la organización escolar. Podríamos decir, los docentes están comprometidos con la actividad y la realidad escolar desde una mirada social "creo que los profesores todos estamos comprometidos con la realidad social y con la actividad escolar".

En este sentido, identificamos que las acciones del posicionamiento docente están vinculadas a la tarea de enseñar constituida con algunos rasgos de la aristocracia profesoral y con la diplomada, cuestionándose, con la tarea de contener a los estudiantes, mediar en su formación como la de apoyar a los padres de éstos, rasgos relacionados con la cuarta posición docente que presentan (Birgin y Pineau, 2014) ante los efectos de la fragmentación educativa. sostiene:

En virtud de lo expuesto, recuperamos un fragmento de Dubet (2010) quien

"el trabajo en las instituciones (...) se desplazan desde las instituciones hacia unos individuos que están menos influidos por el sistema simbólico de un programa. (...) No se trata de que ya no haya más roles, sino de que ya no basta con representar el rol para que el trabajo de socialización se realice. Los individuos deben comprometerse subjetivamente en su trabajo, deben motivarse y motivar a los otros cuando el sistema de motivaciones no es evidente ni unánimemente compartido". (Dubet, 2010, p.24).

\section{6-Bibliografía}

Achilli, E. (1986). La Práctica Docente: Una Interpretación desde los Saberes del Maestro. Buenos. Aires: Cuadernos de Formación Docente. Universidad Nacional de Rosario.

Argentina. Ministerio de Educación, Ciencia y Tecnología de la Nación (2006). Ley de Educación Nacional № 26206/06. Buenos Aires.

Asprella, G. (2013). Parte I: La escuela secundaria, una reflexión sobre sus definiciones. En Pini, M., Mas Rocha, S., Gorostiaga, J., Tello, C. y Asprella, G. (Coord.). La escuela secundaria ¿Modelo en (re) construcción? (pp.17-49). Buenos Aires: Aique. 
Birgin, A. (2006). Pensar la formación de los docentes de nuestro tiempo. En Terigi, F. (comp.). Diez miradas sobre la escuela primaria. (pp.267-294). Buenos Aires: Fundación OSDE/Siglo XXI.

Birgin, A. y Pineau, P. (2015). "Posiciones docentes del profesorado para la enseñanza secundaria en la Argentina: una mirada histórica para pensar el presente". En Revista Teoria e Prática da Educação - "Dossier História da formação docente: olhares latino-americanos". Departamento de Teoria e Prática da Educação da Universidade Estadual de Maringá-UEM, v. 18, núm.1, pp. 47-61.

Dubet, F. (2010). "Crisis de la trasmisión y declive de la institución". En Revista Política y Sociedad, Vol. 47, núm. 2, pp.15-25.

Ezpeleta, J. (2004). "Innovaciones educativas. Reflexiones sobre los contextos en su implementación". En Revista Mexicana de Investigación Educativa, Vol. 9, núm. 21, pp. 403-424.

Ferreyra, H. y Vidales, S. (2012). Cambio educativo para una utopía esperanzadora. En Ferreyra, H. (coord.) Aproximaciones a la educación secundaria en Argentina 20002010: entramados, análisis y propuestas para el debate (pp. 34-58). Córdoba: Comunicarte y Universidad Católica de Córdoba-Facultad de Educación.

Fierro, C., Fortoul, B. y Rosas, L. (1999). Transformando la Práctica Docente. Una propuesta basada en la Investigación Acción. México. Paidós.

Marmissolle, G. (2016). ¿Enseñar? Posibilidades y prácticas docentes en una configuración escolar emergente. En Casenave, G., Errobidart, A., Gallí, M., Marmissolle, G., Martínez Stoessel, P. y Recofsky, M. Trazos de escuela. Un abordaje etnográfico en la educación secundaria obligatoria. (p.p.175-201). Buenos Aires: Miño y Dávila.

Martínez Cerón, G. (2005). Cap. III Sombras y luces de la relación Familia y escuela. En Escudero Muñoz, J., Guarro Pallás A., Matinez Cerón, G., y Riu Sala X. (Ed.) Sistema Educativo y democracia. La escuela del nuevo siglo, 7. Alternativas para un sistema escolar democrático. (pp. 99-149). Madrid, España: Octaedro.

Mendicoa, G. (2003). Sobre tesis y tesistas: lecciones de enseñanza-aprendizaje. Buenos Aires: Espacio.

Morín, E. (1986). El método III. El conocimiento del conocimiento. Madrid: Cátedra.

Ojeda, M. y Saucedo, G. (2015). Las prácticas escolares de participación en la escuela secundaria. Desde el marco normativo a las experiencias y significados que otorgan los actores escolares y las madres a las Jornadas Nacionales Escuela, Familias y Comunidad. En Revista del Instituto de Investigaciones en Educación, Año 6, núm. 6, pp.32-54.

Pineau, P. y Birgin, A. (2006). Esos raros peinados nuevos. ¿Qué traen los futuros docentes? En Feldfeber, M. y Andrade Olivera, D. Políticas educativas y trabajo docente. Nuevas regulaciones ¿Nuevos sujetos? Buenos Aires: Noveduc.

Southwell, M.; Vassiliades, A. (2014). "La noción de posición docente: acerca de la politicidad de la educación". En Revista Educación, Lenguaje y Sociedad Vol. XI, núm.11 pp. 163-187.

Taylor, S. T. y Bogdan, R. (1986). Introducción a los métodos cualitativos de investigación. Buenos Aires: Paidós.

Tiramonti, G. (2003). "Una agenda para el sistema educativo". Revista Novedades Educativas, Año 15, núm. 154. 